\title{
Solo dwarfs III: Exploring the orbital origins of isolated Local Group galaxies with Gaia Data Release 2
}

\author{
Alan W. McConnachie ${ }^{1 \star}$, Clare R. Higgs ${ }^{2}$, Guillaume F. Thomas ${ }^{3}$, Kim A. Venn $^{2}$, Patrick Côté1, \\ Giuseppina Battaglia ${ }^{3,4}$, Geraint F. Lewis ${ }^{5}$. \\ ${ }^{1}$ NRC Herzberg Astronomy \& Astrophysics, 5071 West Saanich Road, Victoria, British Columbia, Canada V9E 2E7 \\ ${ }^{2}$ Physics \& Astronomy Department, University of Victoria, 3800 Finnerty Rd, Victoria, B.C., Canada, V8P 5C2 \\ ${ }^{3}$ Instituto de Astrofísica de Canarias, Calle Via Láctea s/n, E-38206 La Laguna, Tenerife, Spain \\ ${ }^{4}$ Universidad de La Laguna, Avda. Astrofísico Fco. Sánchez, La Laguna, 38200 Tenerife, Spain \\ ${ }^{5}$ Sydney Institute for Astronomy, School of Physics, A28, The University of Sydney, NSW 2006, Australia
}

Accepted XXX. Received YYY; in original form ZZZ

\begin{abstract}
We measure systemic proper motions for distant dwarf galaxies in the Local Group and investigate if these isolated galaxies have ever had an interaction with the Milky Way or M31. We cross-match photometry of isolated, star forming, dwarf galaxies in the Local Group, taken as part of the Solo survey, with astrometric measurements from Gaia Data Release 2. We find that NGC 6822, Leo A, IC 1613 and WLM have sufficient supergiants with reliable astrometry to derive proper motions. An additional three galaxies (Leo T, Eridanus 2 and Phoenix) are close enough that their proper motions have already been derived using red giant branch stars. Systematic errors in Gaia DR2 are significant for NGC 6822, IC 1613 and WLM. We explore the orbits for these galaxies, and conclude that Phoenix, Leo A and WLM are unlikely to have interacted with the Milky Way or M31, unless these large galaxies are very massive $\left(\gtrsim 1.6 \times 10^{12} M_{\odot}\right)$. We rule out a past interaction of NGC 6822 with M31 at $\sim 99.99 \%$ confidence, and find there is a $<10 \%$ chance that NGC 6822 has had an interaction with the Milky Way. We examine the likely origins of NGC 6822 in the periphery of the young Local Group, and note that a future interaction of NGC 6822 with the Milky Way or M31 in the next 4 Gyrs is essentially ruled out. Our measurements indicate that future Gaia data releases will provide good constraints on the interaction history for the majority of these galaxies.
\end{abstract}

\section{INTRODUCTION}

The isolated dwarf galaxies of the Local Group are the ideal systems for understanding the galactic-scale consequences of physical distancing. There seems little doubt that these dwarfs are not currently exposed to the level of interactions that are relatively common-place when orbiting in the more densely populated satellite systems of the Milky Way and M31. What is unclear, however, is if all these galaxies have forever been as isolated as they are now, or if an interaction early in the life of the dwarf caused it to travel to the outskirts of the Local Group in which it is now found.

While there are approximately 100 dwarf galaxies known within the Local Group, most are satellites of the Milky Way or M31. Only about a dozen dwarfs are not clearly associated with one of these two systems, insofar as they are found more than $\sim 300 \mathrm{kpc}$ (the approximate virial radii of the halos of these two galaxies, e.g., Klypin et al. 2002; Posti \& Helmi 2019) from both these large galaxies. It seems unlikely that these dozen galaxies are the totality of all isolated galaxies in the Local Group, and selection effects are likely important. While there has been a concerted efforts to find new satellites of the Milky Way and M31 in recent years (for a list of Milky Way discoveries, see Drlica-Wagner et al. 2020 and references therein; for a list of M31 discoveries, see McConnachie et al. 2018 and references therein), the same is not true for more isolated Local Group members. Such searches are difficult because of their large distances (rendering member stars relatively faint in comparison to Milky Way satellites) and the fact that they could be found anywhere in the sky (in comparison to the relatively small area of sky around M31 in which its satellites are found). To the best of our knowledge, the search for new Local Group dwarf galaxies using the Palomar Observatory Sky Survey and ESO/Science Research Council survey plates by A. Whiting and colleagues remains the most comprehensive search to-date for which completeness estimates are available. This search led to two new galaxies (Antlia and Cetus) out of 206 candidates (Whiting et al. 1997, 1999). Whiting et al. (2007) estimate that their by-eye searches for Local Group dwarfs are essentially complete down to a surface brightness of $26-27$ mags arcsec ${ }^{-2}$ away from the plane of the Milky Way. This surface brightness is comparable to that of Sextans, the lowest surface brightness "classical" satellite of the Milky Way. In this case, Leo T (Irwin et al. 2007) - at a surface brightness of around 25 mags arcsec ${ }^{-2}$ - would be one of only a few galaxies at these surface brightnesses that was not already identified. Clearly, a new search for distant Local Group galaxies (not satellites of M31 and the Milky Way), using the extensive digital sky surveys accumulated since the days of the Palomar Sky Survey, is overdue.

Isolated dwarf galaxies in the Local Group have long been noted to exhibit some general differences to the satellite populations (Einasto et al. 1974). The former generally host younger, bluer, populations, and have significant gas fractions. The latter generally lack significant young populations and have low or negligible gas fractions. Many exceptions to this trend exist (e.g, the LMC, SMC, M33, Cetus, Tucana, Andromeda XVIII). Detailed star formation histories of galaxies throughout the Local Group (e.g., Weisz et al. 2014 and references therein) suggest that in large part the star formation histories 
of both satellites and isolated systems are generally similar over most of cosmic time, and that their star formation histories differ most significantly only in the last gigayear or so. Thus, much of the difference in these populations connects back to the fact that the isolated dwarfs have generally been able to retain their gas to the present day, whereas many satellites have not.

The position-morphology relation that has been identified in the Local Group is seen elsewhere in the Universe (e.g., Bouchard et al. 2009; Geha et al. 2012), and prompts consideration of the influence of nearby large galaxies on the evolutionary paths of dwarfs. Mechanisms at play may include ram pressure stripping (e.g., Mori \& Burkert 2000; Vollmer et al. 2000; Boselli et al. 2014), tidal stripping (e.g., Peñarrubia et al. 2008), tidal stirring (e.g., Mayer et al. 2006; Kazantzidis et al. 2011), induced star formation, and/or strangulation (Kawata \& Mulchaey 2008 and references therein). Comparison of the properties of isolated dwarfs to satellites could therefore shed light on the importance and interplay of several complex physical processes.

An essential element of these considerations is the acquisition of comparable datasets for isolated dwarfs as exist for satellite dwarfs in the Local Group. It is with this in mind that we constructed the Solitary Local (Solo) Dwarfs survey, where our intent is to provide homogeneous, high-quality, wide-field optical characterization of the closest, isolated dwarf galaxies (see Higgs et al. 2016, hereafter Paper I). These dwarfs have been identified based on their current locations, namely that they are more than $300 \mathrm{kpc}$ from either the Milky Way or M31, and are within $3 \mathrm{Mpc}$. The full sample includes, but is not limited to, the Local Group. For that subset that are within the Local Group, an important consideration is whether or not the present positions of these dwarfs are enough to indicate that they have always been isolated.

There are some dwarf galaxies in the Local Group, such as DDO210 or the Sagittarius dwarf irregular galaxy (Sag DIG), that are more than a megaparsec from either of the dominant two-some. In these extreme cases, then barring pathological velocities (which are not implied by their heliocentric radial velocities), there is simply not enough time in the Universe for these galaxies to have previously interacted with M31 or the Milky Way, and then to have reached their current positions (e.g., see Figure 8 of McConnachie 2012). But for systems that are of order $300 \mathrm{kpc}-1 \mathrm{Mpc}$ distant from the large galaxies, it is quite conceivable that they have had a previous interaction (and so potentially been subject to a whole gamut of complex environmental processes), and have since travelled to apocenter far from their host.

Identification of these so-called backsplash systems is important since, unlike truly isolated systems, their properties cannot necessarily be attributed only to secular processes. Early numerical work by Gill et al. (2005) on backsplash galaxies concluded that half of the dwarfs at $1-2 R_{v i r}$ from an $L_{\star}$ galaxy could actually be backsplash systems. More recent work by Teyssier et al. (2012) and Buck et al. (2019) reinforce this finding, and attempt to identify which Local Group dwarfs are most likely backsplash systems based on their positions and/or radial velocities and/or velocity dispersions. Both studies conclude that there is more than a 50\% of NGC6822 and Leo $\mathrm{T}$ having passed close to the Milky Way. Of the remaining isolated Local Group galaxies, Andromeda XXVIII, Cetus, Eridanus 2, IC 1613, Pegasus DIG, Phoenix and Tucana are highlighted as possible backsplash systems (more than a 50\% chance) by one of the studies.

Determination of a galaxy's orbit is the most robust method to identify if it is a backsplash system. Observationally, this requires its position on the sky, its distance, its radial velocity and its proper motion. The first three are all relatively simple measurements. The fourth is not. The first proper motion of a Local Group galaxy that was not in the Milky Way subgroup was made 15 years ago. Brunthaler et al. (2005) identified a water maser in M33, and were able to use the Very Large Baseline Interferometer (VLBI) to obtain a systemic proper motion after correction for M33's rotation. Water masers are only associated with intense star formation, and the most intensely star forming galaxy in the Local Group is the dwarf starburst galaxy IC10. Brunthaler et al. (2007) were able to measure its proper motion using an identical methodology as for M33. M31 itself is a relatively quiescent galaxy in comparison to these two satellites (e.g., Davidge et al. 2012 and references therein), and its proper motion has since been derived from observations with the Hubble Space Telescope (HST; Sohn et al. 2012). Proper motions have also just recently been measured by HST for NGC147 and 185 (Sohn et al. 2020).

The advent of Gaia Data Release 2 (DR2; Gaia Collaboration et al. 2018a) has been a watershed moment for dynamical studies of the Milky Way satellite system, with a large number of papers dedicated to the measurement of almost all the dwarf galaxy satellites of the Milky Way (Gaia Collaboration et al. 2018b; Massari \& Helmi 2018; Simon 2018; Simon et al. 2020; Fritz et al. 2018, 2019; Kallivayalil et al. 2018; Pace \& Li 2019; Longeard et al. 2018, 2020b,a; Mau et al. 2020; McConnachie \& Venn 2020). This sample includes three galaxies - Phoenix, Eridanus II and Leo T - that are in the Solo sample (all located at $\lesssim 450 \mathrm{kpc}$ ). In addition, Gaia DR2 has been used to independently confirm the previous measurements of the proper motions of M31 and M33 (van der Marel et al. 2019), albeit with less accuracy than HST or VLBI. Here, we explore the utility of Gaia data - now and in future data releases - for obtaining the proper motions of the more distant isolated Local Group dwarf galaxies in Solo, to better determine their orbital histories.

Section 2 discusses the datasets that are used in our analysis and the identification of the dwarf galaxies for which we are able to measure proper motions. These measurements are made in Section 3. Section 4 uses these new measurements to explore the orbital parameter space of these galaxies (including analysis for the three dwarf galaxies for which literature estimates of the proper motion are available). Specifically, we examine the likelihood that these systems are backsplash galaxies. Section 5 discusses these findings and summarises our results.

\section{DATA AND QUALITY CONTROL}

Solo is a volume limited, wide field imaging survey of all nearby and isolated dwarf galaxies, and a general introduction to the survey is given in Paper I. Briefly, Solo targets all known dwarf galaxies fainter than the Magellanic Clouds which are within $3 \mathrm{Mpc}$ of the Sun and more than $300 \mathrm{kpc}$ from either M31 or the Milky Way (for a current total of 44 galaxies). Galaxies are observed with either CFHT/Megacam (Boulade et al. 2003) in the northern hemisphere or Magellan/Megacam (McLeod et al. 2015) or IMACS (Dressler et al. 2011) in the southern hemisphere. Some targets are observed with multiple instruments for calibration purposes. The total survey area per galaxy is approximately one square degree, regardless of telescope/instrument (for Magellan, multiple pointings are used to cover this area, whereas only a single pointing is required for CFHT). All dwarfs were observed in the $g-$ and $i$-bands, and some galaxies also have $u$-band (although in what follows we do not use the $u$-band).

Table 1 lists the 16 Solo dwarf galaxies in the Local Group (that is, at or within the zero-velocity survey; see Higgs et al. 2020, hereafter Paper II), ordered by distance modulus. Parameters are taken from 


\begin{tabular}{|c|c|c|c|c|c|c|c|c|}
\hline Galaxy & RA (degs) & Dec (degs) & $(m-M)_{0}(\mathrm{mags})$ & $M_{V}$ (mags) & $v_{h}\left(\mathrm{~km} \mathrm{~s}^{-1}\right)$ & $d_{M W}(\mathrm{kpc})$ & $d_{M 31}(\mathrm{kpc})$ & Recent SF? \\
\hline Phoenix & 27.7762 & -44.4447 & $23.06 \pm 0.12$ & -9.9 & $-21.2 \pm 1.0$ & 409 & 865 & $\mathrm{Y}$ \\
\hline NGC 6822 & 296.2358 & -14.7892 & $23.31 \pm 0.08$ & -15.2 & $-54.5 \pm 1.7$ & 452 & 898 & $\mathrm{Y}$ \\
\hline Andromeda XXVIII & 338.1717 & 31.2161 & $24.1_{-0.2}^{+0.5}$ & -8.5 & $-326.2 \pm 2.7$ & 661 & 368 & $\mathrm{~N}$ \\
\hline IC 1613 & 16.1992 & 2.1178 & $24.39 \pm 0.12$ & -15.2 & $-231.6 \pm 1.2$ & 758 & 520 & $\mathrm{Y}$ \\
\hline Leo A & 149.8604 & 30.7464 & $24.51 \pm 0.12$ & -12.1 & $24.0 \pm 1.5$ & 803 & 1200 & $\mathrm{Y}$ \\
\hline Tucana & 340.4567 & -64.4194 & $24.74 \pm 0.12$ & -9.5 & $194.0 \pm 4.3$ & 883 & 1356 & $\mathrm{~N}$ \\
\hline Peg DIG & 352.1512 & 14.7431 & $24.82 \pm 0.07$ & -12.2 & $-179.5 \pm 1.5$ & 921 & 474 & $\mathrm{Y}$ \\
\hline WLM & 0.4925 & -15.4608 & $24.85 \pm 0.08$ & -14.3 & $-130.0 \pm 1.0$ & 933 & 836 & $\mathrm{Y}$ \\
\hline Sag DIG & 292.4958 & -17.6808 & $25.14 \pm 0.18$ & -11.5 & $-78.5 \pm 1.0$ & 1059 & 1357 & Y \\
\hline Aquarius & 311.7158 & -12.8481 & $25.15 \pm 0.08$ & -10.7 & $-137.7 \pm 2.1$ & 1066 & 1172 & $\mathrm{Y}$ \\
\hline
\end{tabular}

Table 1. All Solo dwarf galaxies in the Local Group and relevant parameters, including their equatorial coordinates, distance moduli, absolute magnitudes, heliocentric velocities and approximate distances from the Milky Way and M31. Parameters are taken from the updated, online compilation from McConnachie (2012). Also indicated is whether the galaxy has any significant recent star formation ( $\lesssim 0.1-1 \mathrm{Gyr}$; see Weisz et al. 2014).

the updated, online compilation from McConnachie (2012) ${ }^{1}$. Their distances from the center of the Milky Way and from M31 are also given in Table 1 assuming that the Sun is at a distance of $8.122 \mathrm{kpc}$ (Gravity Collaboration et al. 2018) from the Galactic Center, and that M31 is at a distance of $783 \mathrm{kpc}$ (McConnachie et al. 2005). For each of the galaxies in Table 1, we have wide-field, ground-based photometry, the majority of which was presented in Paper II. The reader is referred to Papers I and II for details of all data acquisition, reduction and processing.

For the three closest galaxies (Eridanus 2, Phoenix and Leo T), the brightest red giant branch stars in these systems are luminous enough to have reliable astrometry in Gaia DR2, and proper motions for these three systems have previously been measured (Fritz et al. 2018; Pace \& Li 2019; McConnachie \& Venn 2020, hereafter MV2020). For the remaining systems, the tip of the RGB is fainter than the Gaia DR2 detection limit. For those galaxies with only old or intermediate-age stellar populations - specifically Andromeda XXVIII, Cetus, Andromeda XXXIII (Perseus), Tucana, and Andromeda XVIII - none of their stars are bright enough to have reliable astrometry in Gaia DR2. However, for those galaxies which also have recent star formation, it is possible for both blue and red supergiants to be (significantly) more luminous than the tip of the RGB, depending on their mass and age (e.g., see the original spectroscopic studies of supergiants in NGC 6822 and WLM by Venn et al. 2001, 2003). Indeed, this trait has allowed the determination of the proper motions of M31 and M33 using Gaia DR2 (van der Marel et al. 2019), despite both galaxies being located at approximately $800 \mathrm{kpc}$ from the Milky Way.

For the 8 galaxies in Table 1 which have some evidence of younger stellar populations (e.g., see Paper II, Weisz et al. 2014) and which do not already have derived proper motions, we cross-match our ground-based photometry to Gaia DR2. Specifically, we only select those cataloged objects that are classified as stellar (i.e., within $2-\sigma$ of the locus of point sources) in each of the $g$ and $i$-bands. In all cases, sources are matched to within a few tenths of an arcsecond or better. For the Gaia parameters, we only consider stars with full 5 parameter astrometric solutions, and high quality astrometry as defined via the

\footnotetext{
1 http://www.astro.uvic.ca/ $\sim$ alan/Nearby_Dwarf_Database. html
}

renormalised unit weighted error (ruwe; see Lindegren et al. 2018 and discussion in the Gaia DR2 Documentation Release 1.2). We adopt ruwe $<1.4$. We also only consider stars whose parallaxes are consistent with them being located at a similar distance to the galaxy. That is, the $3-\sigma$ parallax range measured by Gaia DR2 must overlap the $3-\sigma$ parallax range implied by the distance modulus of the dwarf as given in Table 1. We correct for the global zero-point of the parallax in Gaia DR2 of -0.029mas (Lindegren et al. 2018).

The ground-based photometry is presented in Figure 1. Here, the left panel of each pair of plots shows the ground-based CMD for the inner regions of each field centered on each galaxy. The right panel of each pair shows those sources that are also in Gaia DR2, and which pass the cuts described above. All photometry is extinctioncorrected with the reddening maps from Schlegel et al. (1998) using the python interface dustmaps (Green 2018). We use the extinction coefficients derived using the Padova isochrone (Girardi et al. 2002) web-tool ${ }^{2}, A_{g}=1.191 \times A_{V}$ and $A_{i}=0.854 \times A_{V}$, where $A_{V}=$ $2.742 \times E(B-V)$ (Schlafly \& Finkbeiner 2011).

In contrast to MV2020, we do not apply any cuts to the Gaia DR2 data based on the quality of the Gaia photometry, since we rely instead on the ground-based data for our photometric selection. This is an important point: experimentation with the Gaia DR2 photometry shows that the formal quality of the Gaia photometry for many of the sources being examined is quite poor (as quantified via the fluxexcess parameter; see Lindegren et al. 2018). We expect this is to do with the relatively high degree of crowding in these distant dwarf galaxies (see also discussion of this point in van der Marel et al. 2019 as it relates to the centers of M31 and M33). Of course, the ground-based data can also be crowded in the very central regions of the dwarfs, especially at faint magnitudes. However, the generally good or excellent image quality of our ground-based data, in multiple bands, combined with the relative brightness of the sources under consideration (with respect to the limiting magnitude of the ground-based data) means that the photometric quality of Gaia DR2 is not a limiting factor for this analysis, so long as the astrometric quality of the data is sufficient (as parameterized by ruwe).

For the 8 galaxies in Figure 1, blue supergiants are easily visible

2 http://stev.oapd.inaf.it/ 

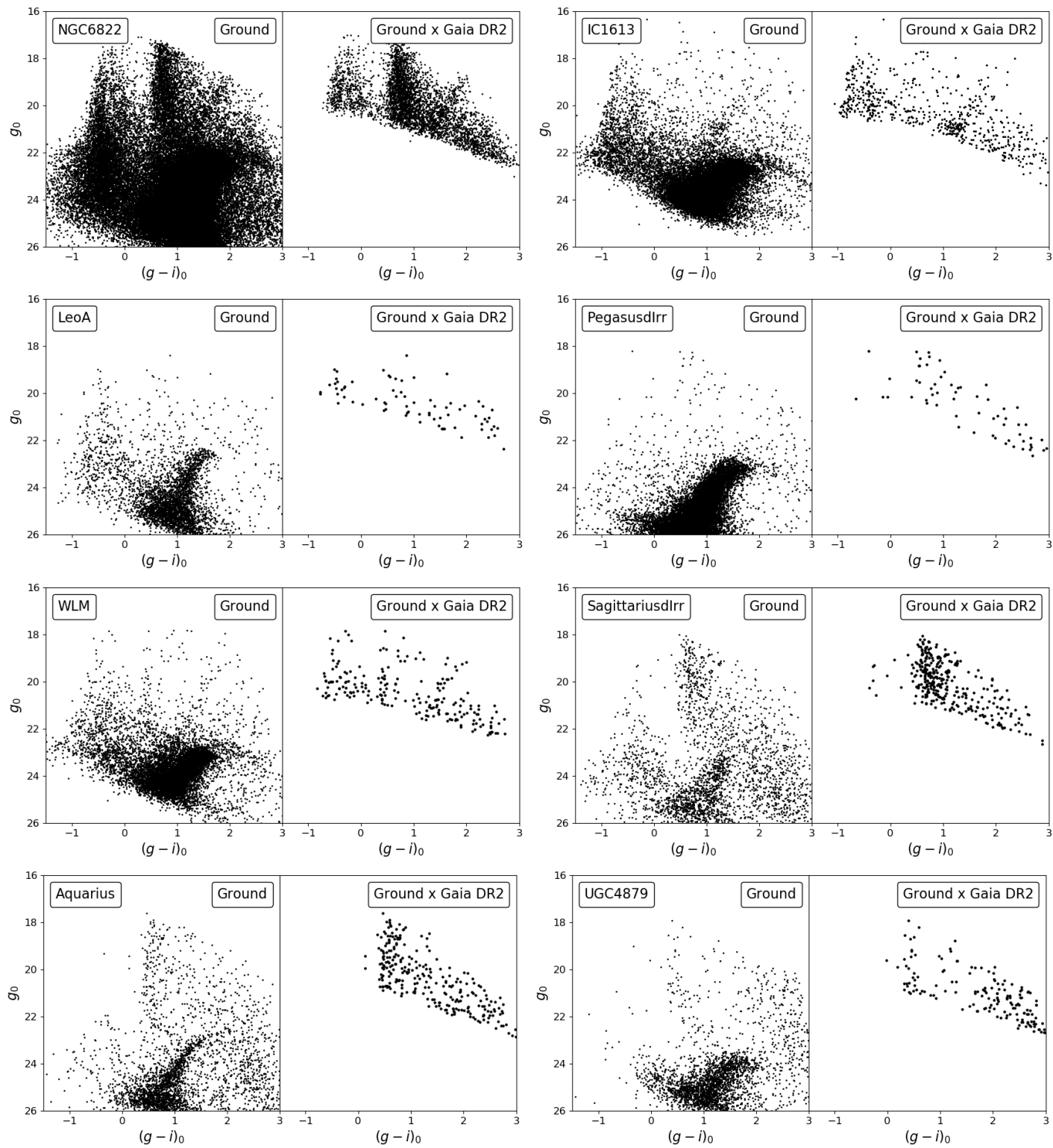

Figure 1. Extinction corrected, ground-based colour magnitude diagrams (CMDs) for each of the galaxies listed in Table 1 that do not already have Gaia DR2 systemic proper motions, but which have some evidence of younger stellar populations. The left panel of each pair of CMDs shows the ground-based CMDs for the inner regions of each field centered on each galaxy. The right panel of each pair shows those sources that are also in Gaia DR2, and which pass the quality cuts described in the text.

as a broad vertical plume of stars generally blueward of $(g-i)_{0}=0$ in the left panels (although UGC 4879 in particular does not have many such stars; see also Jacobs et al. 2011). In all cases, they extend to luminosities brighter than the tip of the RGB. However, not all galaxies have many of these stars bright enough to have robust astrometry in Gaia DR2. Specifically, Peg DIG, Sag DIG, Aquarius and UGC 4879 do not have more than five or six candidate blue supergiants (if any) that remain in the right panels of Figure 1. In addition to blue supergiants, red supergiants are clearly visible in NGC 6822, IC 1613 and WLM, and form a diagonal sequence at a colour of around $(g-i)_{0} \simeq 1$ extending in the direction of the top-right corner. Equivalent sequences are not clearly visible in any of the other galaxies. Leo A is a marginal case with respect to the other systems since blue supergiants are present in the Gaia DR2 data but there is no clear red supergiant sequence.
The right panels of Figure 1 suggest that systemic proper motions should be able to be derived using Gaia DR2 data for NGC 6822, IC 1613, Leo A and WLM. We make these measurements in Section 3.

\section{MEASUREMENT OF PROPER MOTIONS}

\subsection{Method}

We adopt a similar methodology to MV2020, and we refer the reader to Section 3 of that paper for a full description of the method. In brief, we seek to maximize the likelihood of the data, where the total likelihood is given by

$\mathcal{L}=f_{\text {dwarf }} \mathcal{L}_{\text {dwarf }}+\left(1-f_{\text {dwarf }}\right) \mathcal{L}_{M W}$ 

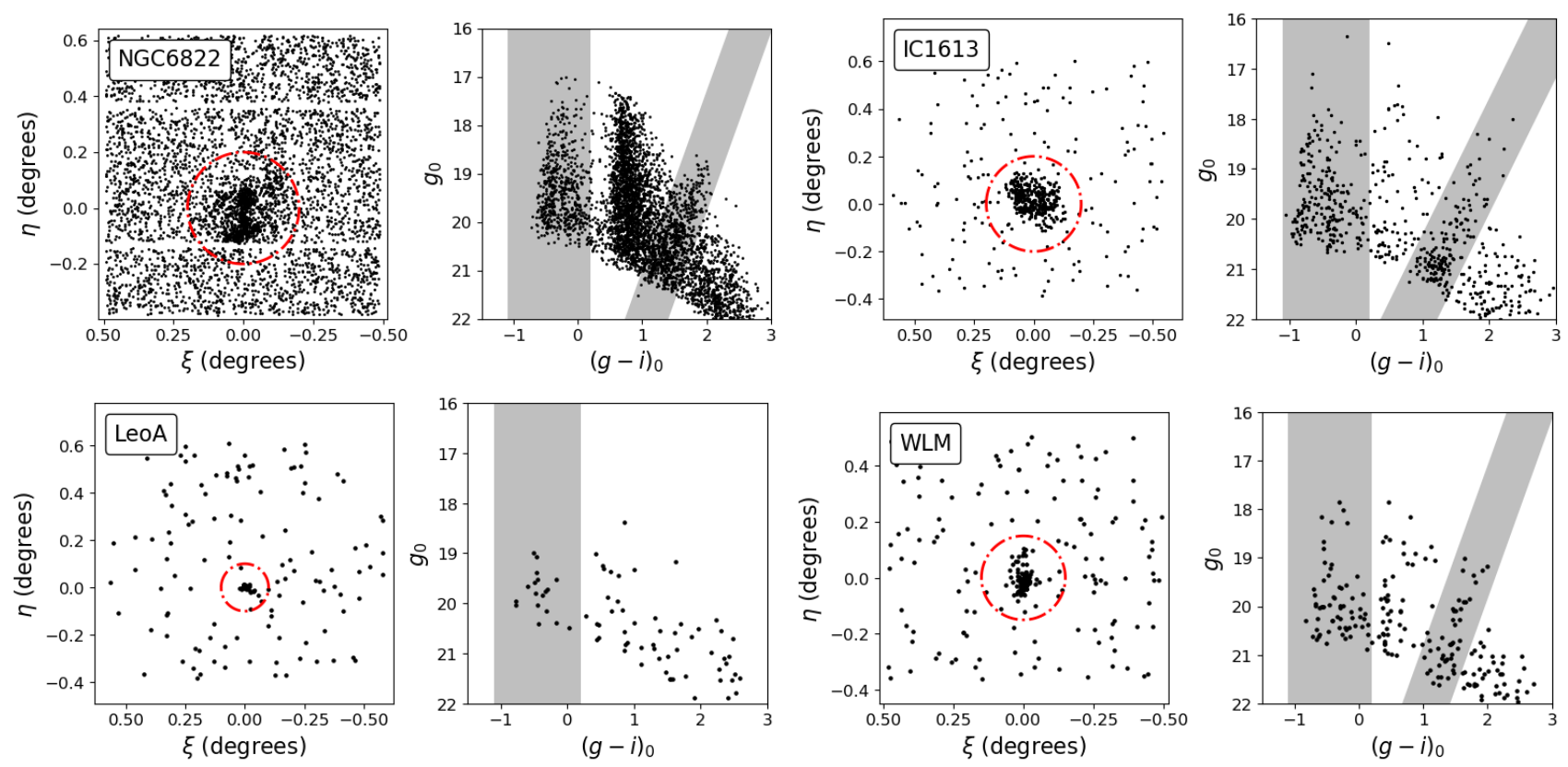

Figure 2. The spatial and colour-magnitude distributions of Gaia DR2 sources in each field surrounding NGC 6822, IC 1613, Leo A and WLM. The left panels show the distribution in the tangent plane of all Gaia DR2 sources that lie within the shaded regions (blue and red supergiant regions) of the CMD in the right panels. The right panel shows the CMD of all Gaia DR2 sources that lie within the dashed red circles in the left panels.

where $\mathcal{L}_{\text {dwarf }}$ and $\mathcal{L}_{M W}$ are the likelihoods for the dwarf galaxy and MW foreground, respectively, and $f_{\text {dwarf }}$ is the fraction of stars in the satellite. $\mathcal{L}_{\text {dwarf }}$ can be broken down as

$\mathcal{L}_{\text {dwarf }}=\mathcal{L}_{S} \mathcal{L}_{C M} \mathcal{L}_{P M}$

where $\mathcal{L}_{S}, \mathcal{L}_{C M}$ and $\mathcal{L}_{P M}$ are the likelihoods from the spatial information, colour-magnitude information, and proper motion information, respectively. $\mathcal{L}_{M W}$ can similarly be broken down into the product of its three constituent likelihoods.

We therefore need to define likelihood functions for the spatial and colour-magnitude distribution of sources in the dwarf. We also need to define spatial, colour-magnitude and proper motion likelihood functions for the Milky Way foreground population. Using these, we find the systemic proper motion of the dwarf that maximises the probability of the data given these models, with the adoption of suitable priors on the unknown parameters $\left(\mu_{\alpha} \cos \delta, \mu_{\delta}\right)$ dwarf and $f_{\text {dwarf. }}$.

Figure 2 shows the spatial and colour-magnitude distributions of Gaia DR2 sources in each field surrounding NGC 6822, IC 1613, Leo A and WLM. Specifically, the left panels show the distribution in the tangent plane of all Gaia DR2 sources that lie within the shaded regions of the CMD in the right panels. The right panel shows the CMD of all Gaia DR2 sources that lie within the dashed red circles in the left panels. The grey regions are defined empirically to be simple polygons that enclose the blue and red supergiants in each galaxy. For Leo A, we only consider blue supergiants due to the absence of any clear red supergiant sequence. For the blue supergiants, the red edge of the selection box is placed bluer than the edge of the Milky Way foreground (that is delineated by the main sequence turn-off of stars around $(g-i)_{0} \sim 0.4$ at a range of distances in the halo of the Milky Way).

For the spatial, colour-magnitude and proper motion likelihood functions for the Milky Way foreground population, the corresponding likelihood functions are defined in exactly the same way as in
MV2020. That is, for the spatial likelihood function, we consider the contamination to be uniform across the field. For the colourmagnitude and proper motion likelihood functions, we use the relevant distributions of stars that are outside of the red dashed circles in Figure 2. These are converted into density maps and normalized, as described in MV2020.

For the spatial likelihood function of the satellite, MV2020 used 2D-lookup maps based on the parameterized structure of the galaxies as reported in the literature. However, in general these parameters are derived from the distribution of old and intermediate age stellar populations (usually upper main sequence and/or red giant branch stars; e.g. Muñoz et al. 2018), and these can have a very different spatial distribution to the young stars that are the focus here (for example, note the irregular distribution of young stars in NGC 6822). In the absence of good parameterizations of the spatial distribution of young stars, we instead build a likelihood function based on the projected density distribution of candidate blue supergiants. A density map ( 0.1 arcmin pixels) is produced using stars that lie within the grey regions corresponding to the blue supergiant locus for each galaxy, and that are within the dashed red circles in Figure 2. These density distributions are then convolved with a Gaussian with a standard deviation of 0.5 arcmins, and normalized appropriately. We rely on blue supergiants to define the spatial likelihood function because they can be identified with almost no contamination from foreground populations. The red supergiants in NGC6822, IC1613 and WLM are assumed to be spatially distributed in much the same way as the blue supergiants, which seems reasonable

For the CMD likelihood function of the satellite, MV2020 used 2D-lookup maps based on the expected location of upper main sequence, sub-giant and red giant branch stars as given by isochrones with appropriate ages and metallicities. In comparison, for blue and red supergiants, the expected CMD distribution is not as sharply defined and they are more difficult to model without reasonably detailed recent star formation histories (e.g., see the beautiful reconstruction of the star formation history of Sextans A using blue and red super- 
giants by Dohm-Palmer et al. 2002). Instead, we instead use a simple, empirical model of the expected CMDs, which is that the relevant stars are expected to lie somewhere in the grey regions of the CMDs in Figure 2. A uniform likelihood is assumed over these regions.

In MV2020, two different sets of priors were imposed on the systemic proper motion of the dwarf. The first was that the tangential velocity could not be unrealistically high compared to the Milky Way; the second favored systemic motions where stars that appeared to be radial velocity members were more likely to be assigned membership. In the current analysis, there are no radial velocities to consider, and we do not have any expectation on the tangential velocity of the galaxy with respect to the Milky Way. As such, the prior requires only that the systemic proper motion in each direction is less than 10 mas/yr, with a uniform likelihood over this range. We adopt a uniform prior for $f_{\text {dwarf }}$ in the interval $0 \leq f_{\text {dwarf }} \leq 1$, the same as MV2020.

\subsection{Results}

For NGC 6822, IC 1613, Leo A and WLM, we construct the spatial, colour-magnitude and proper motion likelihood maps as described in the previous section and explore parameter space using emcee (Foreman-Mackey et al. 2013, 2019) for those values of $f_{\text {dwarf }}$ and $\left(\mu_{\alpha} \cos \delta, \mu_{\delta}\right)$ dwarf that maximise the probability of the data.

Table 2 lists the resulting median values and 16th and 84th percentiles for the three unknown parameters for each of our four galaxies. We also list the proper motions for Eridanus 2, Phoenix and Leo $\mathrm{T}$, as derived in MV2020 ${ }^{3}$. We also list the corresponding Galactocentric tangential velocity components assuming $\left(R_{\odot}, V_{c}\right)=\left(8.122 \mathrm{kpc}, 229 \mathrm{~km} \mathrm{~s}^{-1}\right)$ and $\left(U_{\odot}, V_{\odot}, W_{\odot}\right)=$ $(11.1,12.24,7.25) \mathrm{km} \mathrm{s}^{-1}$ (Gravity Collaboration et al. 2018; Schönrich et al. 2010), for the distance moduli given in Table 1.

All error bars in Table 2 describe random errors only, and systematic uncertainties in the proper motions are not included. Gaia Collaboration et al. (2018b) analyse the systematic uncertainties in the proper motions of stars in the Sagitarrius dwarf spheroidal using Gaia DR2, and conclude that on spatial scales of around 0.2 degrees (the approximate spatial scale of the supergiant distributions in NGC 6822 and IC 1613), the systematic uncertainty is $\sim 0.035$ mas/year. Lindegren et al. (2018) analyse the spatial covariance of the proper motions of quasars, and conclude that on scales less than 0.125 degrees (the approximate spatial scales of all the other galaxies in Table 2, excluding NGC 6822 and IC 1613), the systematic uncertainty is $\sim 0.066 \mathrm{mas} / \mathrm{yr}$. It is worth emphasising the scale of these uncertainties for the galaxies under consideration. At the distances of NGC 6822 (462 kpc) and WLM (933 kpc), a systematic uncertainty of [0.035/0.066] mas/year corresponds to an uncertainty in the tangential velocity of these dwarfs of $[73 / 138] \mathrm{km} \mathrm{s}^{-1}$ and $[148 / 280] \mathrm{km} \mathrm{s}^{-1}$, respectively, in each direction. As a reference value, a simple estimate of the expected velocity dispersion of the Local Group gives $\sigma_{L G} \simeq 161\left(\frac{M_{L G}}{3 \times 10^{12} M_{\odot}}\right)^{\frac{1}{2}}\left(\frac{500 k p c}{R_{L G}}\right)^{\frac{1}{2}} \mathrm{~km} \mathrm{~s}^{-1}$.

Figure 3 shows the spatial, colour-magnitude, and proper motion distributions for member stars in NGC 6822, IC 1613, Leo A and

\footnotetext{
${ }^{3}$ Note that the relative foreground for these three galaxies is much higher than that for the dwarf irregulars studied here due to differences in the tracer population that is used (RGB stars versus supergiants). This results in very different values for $f_{\text {dwarf }}$ between the two studies.
}

WLM (magenta stars). Here, we use the fact that the probability of any star being a member of the dwarf is given by

$P_{\text {dwarf }}=\frac{f_{\text {dwarf }} \mathcal{L}_{\text {dwarf }}}{f_{\text {dwarf }} \mathcal{L}_{\text {dwarf }}+\left(1-f_{\text {dwarf }}\right) \mathcal{L}_{M W}}$.

We define members as those stars with $P_{\text {dwarf }} \geq 0.5$. Inspection of Figure 3 shows that the probable members of these galaxies appear to cluster appropriately in all three parameter spaces, although the very few stars in Leo A mean that there is not as obvious a cluster of points in proper motion space as for the other three galaxies.

\section{ORBITAL HISTORIES}

Here, we consider the orbits of our target dwarf galaxies within the Local Group to better understand what constraints the newly derived systemic proper motions place on their orbital histories. In particular, we examine the likelihood that these dwarfs have ever passed within $300 \mathrm{kpc}$ of either M31 or the Milky Way.

\subsection{The Local Group potential}

We explore the orbits of each of the dwarf galaxies backwards in time within the Local Group. All our orbital calculations use gyrfalcON (Dehnen 2000). Our intent is to determine only if the dwarf has passed within $300 \mathrm{kpc}$ of either the Milky Way or M31. Each of the dwarf galaxies starts well outside the virial radius of either the Milky Way or M31: $R_{M W, v i r} \sim 287 \mathrm{kpc}$ assuming an NFW profile with a total mass of $M_{M W, v i r} \simeq 1.3 \times 10^{12} M_{\odot}$ (Posti \& Helmi 2019), and this should be compared to the present-day distances of the closest dwarfs (for the MW - Eridanus 2, $d_{M W} \simeq 382 \mathrm{kpc}$; for M31 - IC $1613, d_{M W} \simeq 520 \mathrm{kpc}$ ). As such, we model each large galaxy as a point mass. gyrfalcON does not allow for the use of tracer particles, so we instead set the mass of the dwarf to be tiny in comparison to either of the two large galaxies.

We adopt the distance modulus of M31 to be $d_{\text {mod }}=24.47 \pm 0.07$ (McConnachie et al. 2005) and its heliocentric radial velocity to be $v_{h}=-301 \pm 1 \mathrm{~km} \mathrm{~s}^{-1}$ (van den Bergh 2000). We adopt the systemic proper motion of M31 derived using Hubble Space Telescope observations (Sohn et al. 2012) as given in van der Marel et al. (2012a), namely $\mu_{\alpha} \cos \delta=0.045 \pm 0.013$ mas/year, $\mu_{\delta}=-0.032 \pm 0.012$ mas/year. For the dwarfs, we use the distance moduli and heliocentric radial velocities as given in Table 1 , and the systemic proper motions as given in Table 2. We add in quadrature a systemic proper motion uncertainty to the random uncertainties given in this table. For NGC 6822 and IC 1613, we use $\sigma_{\text {sys }}=0.035$ mas/year (Gaia Collaboration et al. 2018b) given the relatively large angular sizes of these system. For the remaining galaxies, we use $\sigma_{s y s}=0.066$ mas/year (Lindegren et al. 2018). We transform all velocities and proper motions to a Galactocentric frame using the parameters given in Section 3.2.

There is still considerable debate over the total masses of the Milky Way and M31. The former offers more opportunity for study, and Eadie \& Jurić (2019) note that there is reasonable agreement in the literature regarding the mass at small $(\lesssim 40 \mathrm{kpc}$ ) radius. More difficult to constrain is the mass at large radius, where there are fewer tracers and generally poorer data, and many estimates rely on extrapolation of results obtained at smaller radius, given some model assumptions. Bland-Hawthorn \& Gerhard (2016) provide a good review of the relevant literature, and calculate the average of a range of studies based on analysis of the kinematics of the stellar halo. They 


\begin{tabular}{|c|c|c|c|c|c|c|c|c|}
\hline Galaxy & $\mu_{\alpha} \cos \delta(\mathrm{mas} / \mathrm{yr})$ & $\mu_{\delta}(\mathrm{mas} / \mathrm{yr})$ & $f_{\text {dwarf }}$ & $v_{\alpha} \cos \delta\left(\mathrm{km} \mathrm{s}^{-1}\right)$ & $v_{\delta}\left(\mathrm{km} \mathrm{s}^{-1}\right)$ & $v_{t}\left(\mathrm{~km} \mathrm{~s}^{-1}\right)$ & $v_{r}\left(\mathrm{~km} \mathrm{~s}^{-1}\right)$ & Source \\
\hline Leo $\mathrm{T}$ & $0.10_{-0.69}^{+0.67}$ & $0.01_{-0.56}^{+0.57}$ & $0.002 \pm 0.001$ & $227_{-1364}^{+1324}$ & $237_{-1107}^{+1126}$ & 328 & -63 & MV2020 \\
\hline IC 1613 & $0.08^{+0.06}$ & $-0.04 \pm 0.03$ & $0.96 \pm 0.01$ & $141_{-251}^{+215}$ & $32 \pm 107$ & 145 & -150 & This paper \\
\hline Leo A & $0.49 \pm 0.32$ & $-0.46 \pm 0.28$ & $0.96^{+0.03}$ & $1900 \pm 1211$ & $-1507 \pm 1059$ & 2425 & -20 & This paper \\
\hline WLM & $0.11 \pm 0.08$ & $-0.10 \pm 0.05$ & $0.93^{+0.02}$ & $356 \pm 346$ & $-231 \pm 216$ & 425 & -70 & This paper \\
\hline
\end{tabular}

Table 2. Median, 16th and 84th percentiles of the probability density functions for the three unknown parameters for all Solo dwarf galaxies in the Local Group for which derivation of a systemic proper motion is currently possible (Table 4 of MV2020 presents values for Eridanus 2, Leo T and Phoenix; the current paper derives values for NGC 6822, IC 1613 and WLM using a modification of the same method). The corresponding tangential velocity components in a Galactocentric frame of reference are listed, as well as the overall tangential velocity $\left(\mathrm{v}_{t}\right)$. The implied Galactocentric radial velocities are listed in the last column for comparison, and are converted from the heliocentric radial velocities listed in Table 1 (see text for details).
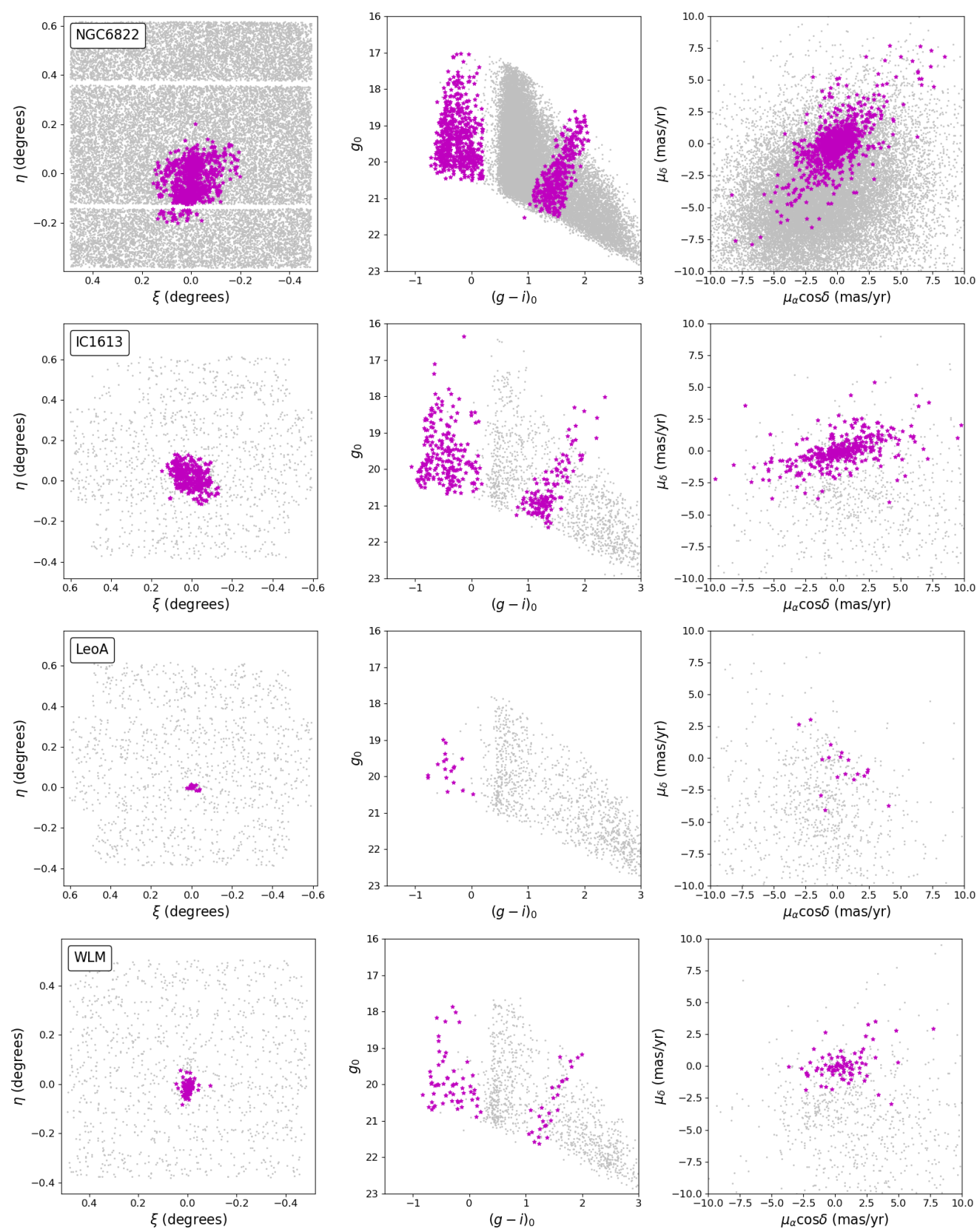

Figure 3. The spatial (left panels), colour-magnitude (middle panels), and proper motion (right panels) distributions for member stars (magenta stars) in NGC 6822, IC 1613, Leo A and WLM. Grey points show all Gaia DR2 sources that pass our quality-control criteria described in the text. 
find that $M_{M W} \simeq(1.3 \pm 0.3) \times 10^{12} M_{\odot}$. This is an identical value to that obtained by Posti \& Helmi (2019), on extrapolation of the mass that they measure within $20 \mathrm{kpc}$, based upon Gaia DR2 kinematics of globular clusters. A different analysis of globular cluster data - including but not limited to Gaia DR2 - by Eadie \& Jurić (2019) favors a slightly lighter mass, $M_{M W} \simeq\left(0.7_{-0.19}^{+0.4}\right) \times 10^{12} M_{\odot}$ (where the uncertainties describe the $95 \%$ credible intervals). Cautun et al. (2020) estimate $M_{M W}=\left(1.08_{-0.14}^{+0.20}\right) \times 10^{12} M_{\odot}$ from analysis of the Gaia DR2 rotation curve and other data.

For M31, its integrated properties generally suggest that it is more massive than the MW (e.g, Hammer et al. 2007). Watkins et al. (2010) analysed the satellite kinematics and determined that $M_{M 31}=(1.4 \pm 0.4) \times 10^{12} M_{\odot}$. Fardal et al. $(2013)$ examined the dynamics of the Giant Stellar Stream (Ibata et al. 2005) and concluded that $M_{M 31} \simeq(1.9 \pm 0.4) \times 10^{12} M_{\odot}$. Patel et al. (2017) combine astrometric studies with cosmological simulations to estimate that $M_{M 31}=\left(1.37_{-0.75}^{+1.39}\right) \times 10^{12} M_{\odot}$ (where they also estimate that $\left.M_{M W}=\left(1.02_{-0.55}^{+0.77}\right) \times 10^{12} M_{\odot}\right)$. A direct measurement of the mass ratio between M31 and the MW was made by Peñarrubia et al. (2014), who found that $f=M_{M W} / M_{M 31}=0.54_{-0.17}^{+0.23}$ by analysis of the effect of the Local Group mass on the Hubble expansion of its nearest neighbours.

\subsection{Exploring parameter space}

Here, we run a suite of simulations for each dwarf in which we run their orbits back in time, varying their two tangential velocity components across a grid, to determine which combinations yield interactions with the Milky Way and/or M31. We then compare this parameter space to the actual measured proper motions of the dwarfs.

We vary the Galactocentric tangential velocity of each dwarf in the range $-1000 \mathrm{~km} \mathrm{~s}^{-1} \leq v_{\alpha} \cos \delta, v_{\delta} \leq 1000 \mathrm{~km} \mathrm{~s}^{-1}$, searching the full grid of values in $5 \mathrm{~km} \mathrm{~s}^{-1}$ increments. We note that the velocity dispersion of the Local Group is expected to be of or$\operatorname{der} \sigma_{L G} \simeq 161\left(\frac{M_{L G}}{3 \times 10^{12} M_{\odot}}\right)^{\frac{1}{2}}\left(\frac{500 k p c}{R_{L G}}\right)^{\frac{1}{2}} \mathrm{~km} \mathrm{~s}^{-1}$, and so this grid search extends out to velocities that would certainly make these galaxies unbound to the Local Group. All other positional and dynamical parameters of the dwarf and M31 are fixed to their preferred values as described above. For the masses of the Milky Way and M31, we use three different combinations:

(i) LG1: $M_{M W}=1.3 \times 10^{12} M_{\odot}, f=1.0$;

(ii) LG2: $M_{M W}=1.3 \times 10^{12} M_{\odot}, f=0.5$;

(iii) LG3: $M_{M W}=1.6 \times 10^{12} M_{\odot}, f=1.0$.

Each of these LG realisations uses a mass for the MW that is either somewhat "average" or to the upper end of the recent estimates (see the discussion above). We purposefully explore orbits using these large masses because, in what follows, the most conservative estimates on the chance of an interaction between a dwarf and the MW/M31 will be obtained under the assumption of larger masses (if an interaction is not going to occur assuming a larger mass estimate, then it will definitely not happen for a lighter mass, all other things being equal).

The point mass approximation for M31 and the MW is not sufficient to determine exact pericenters for the dwarf orbits. However, we only seek to determine if the dwarf passes within $300 \mathrm{kpc}$ of either the Milky Way or M31 (the approximate virial radius of these galaxies). Prior to reaching this distance from the Milky Way or M31, the dwarf will have been affected by the gravitational influence of essentially the total mass of the galaxy, thus the point mass approximation is sufficient up to this point. We do not seek to make any statements about what happens to the orbit after this threshold is reached.

Figures 4 and 5 show the results of these simulations for each dwarf. The different panels for each dwarf correspond to the LG1, LG2 and LG3 setups (left to right, respectively). Red dashed lines delineate the regions of parameter space where the orbits bring the dwarf within $300 \mathrm{kpc}$ of the Milky Way. Blue dotted lines delineate the regions of parameter space where the orbits bring the dwarf within $300 \mathrm{kpc}$ of M31. If no dotted or dashed lines are present, there is no region of parameter space that brings the dwarf within $300 \mathrm{kpc}$ of these galaxies. Overlaid on each panel is the measured proper motion of the dwarf as given in Table 2. Black lines show random errors, and the grey lines also include the systematic uncertainties described earlier.

For the 5 galaxies that are currently closer to the Milky Way than M31 - Eridanus 2, Phoenix, Leo T, NGC 6822 and Leo A - there is a region of parameter space centered around a tangential velocity of zero in which these galaxies could have had an interaction with the Milky Way. However, in the cases of Phoenix and Leo A, these only exist for a more massive Milky Way. Even here, any tangential velocity in excess of $35-40 \mathrm{~km} \mathrm{~s}^{-1}$ or so for each dwarf would move the galaxy into the family of orbits which have not had an interaction.

For WLM and IC 1613, no solutions are found in which they have interacted with the Milky Way. For WLM, it is only possible for it to have interacted with M31 in the scenarios where M31 is relatively massive. There is a reasonably large region of parameter space in which IC 1613 could have interacted with M31.

It is interesting to note that NGC 6822, Leo T and Leo A could have interacted with either the Milky Way or M31, depending on their tangential velocities. For the latter two, M31 interactions can only have occured in a relatively small region of parameter space for the setup with the most massive M31 $\left(\sim 2.6 \times 10^{12} M_{\odot}\right)$. For NGC 6822 , there is a reasonably large area of parameter space for M31 or Milky Way interactions for all three setups.

Figures 4 and 5 provide a simple context to understand the usefulness, or otherwise, of the current proper motion measurements for each of the dwarfs:

- Eridanus 2: This dwarf can only have passed within $300 \mathrm{kpc}$ of the Milky Way if its Galactocentric tangential velocity is less than $\sim 90 \mathrm{~km} \mathrm{~s}^{-1}$. This is at the $1-\sigma$ edge of the current proper motion measurement. The allowable parameter space for a backsplash solution is consistent with a similar study by Blaña et al. (2020), who consider the orbits of Eridanus 2, Phoenix, Leo T and Cetus;

- Phoenix: Of the explored setups, the only way for Phoenix to have ever passed with $300 \mathrm{kpc}$ of the Milky Way if it has a small $\left(\lesssim 35 \mathrm{~km} \mathrm{~s}^{-1}\right.$ ) tangential velocity and the total mass of the Milky Way is relatively massive (as in LG3; at the upper end of the mass range to which it has been constrained by recent measurements). This is also consistent with the study by Blaña et al. (2020). If the Milky Way is really this massive, then the uncertainties on the current proper motion are currently a factor of a few too large to distinguish a backsplash scenario from a more isolated orbit. As Phoenix requires the Milky Way to be massive in order for an interaction to occur, tightening constraints on the Milky Way's total mass will likely prove valuble in definitely ruling out a backsplash origin for Phoenix;

- Leo T: If Leo T has a tangential velocity less than $\sim 80 \mathrm{~km} \mathrm{~s}^{-1}$, then it is probably a Milky Way backsplash system, as also concluded by Blaña et al. (2020). Interestingly, in the case of a very massive M31 (as in LG2), there is also a small region of parameter space in which Leo $\mathrm{T}$ could be a M31 backsplash system (this is also the case for Leo A; see later). However, the current proper motion measurement 

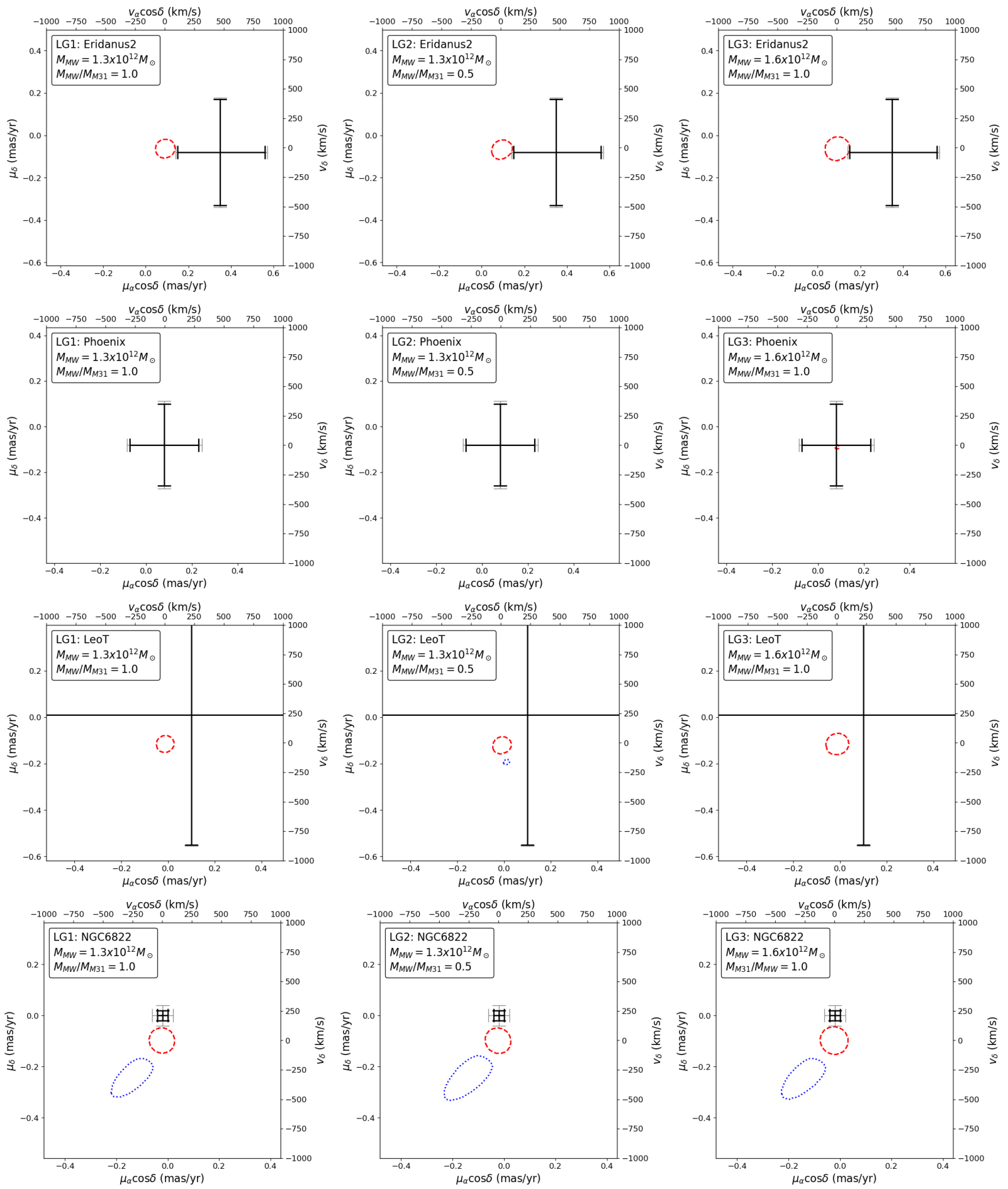

Figure 4. Results of the search through orbital parameter space for Eridanus 2, Phoenix, Leo T and NGC 6822. The different panels for each dwarf correspond to the LG1, LG2 and LG3 setups (left to right, respectively; see text for details). Red dashed lines delineate the regions of parameter space where the orbits bring the dwarf within $300 \mathrm{kpc}$ of the Milky Way. Blue dotted lines delineate the regions of parameter space where the orbits bring the dwarf within $300 \mathrm{kpc}$ of M31. If no dotted or dashed lines are present, it means there is no region of parameter space that brings the dwarf within $300 \mathrm{kpc}$ of these galaxies. Overlaid on each panel is the measured proper motion of the dwarf as given in Table 2. Black lines show random errors, and grey lines also include the systematic uncertainties.

is utterly useless for providing any meaningful constraints in this respect. It is worth noting that the member stars of Leo $\mathrm{T}$ (and to a lesser extent, Leo A) have not been observed as frequently as in other dwarf galaxies as a result of the Gaia scanning law. In particular, the average number of "along-scan" measurements by the spacecraft for the member stars of Leo $\mathrm{T}$ is astrometric_n_obs_al $\simeq 100$ (astrometric_n_obs_al $\simeq 127$ for Leo A). For comparison, NGC 6822 and IC 1613 have around 50\% more measurements on average ( $150-170)$, WLM and Eridanus 2 have about twice as many 

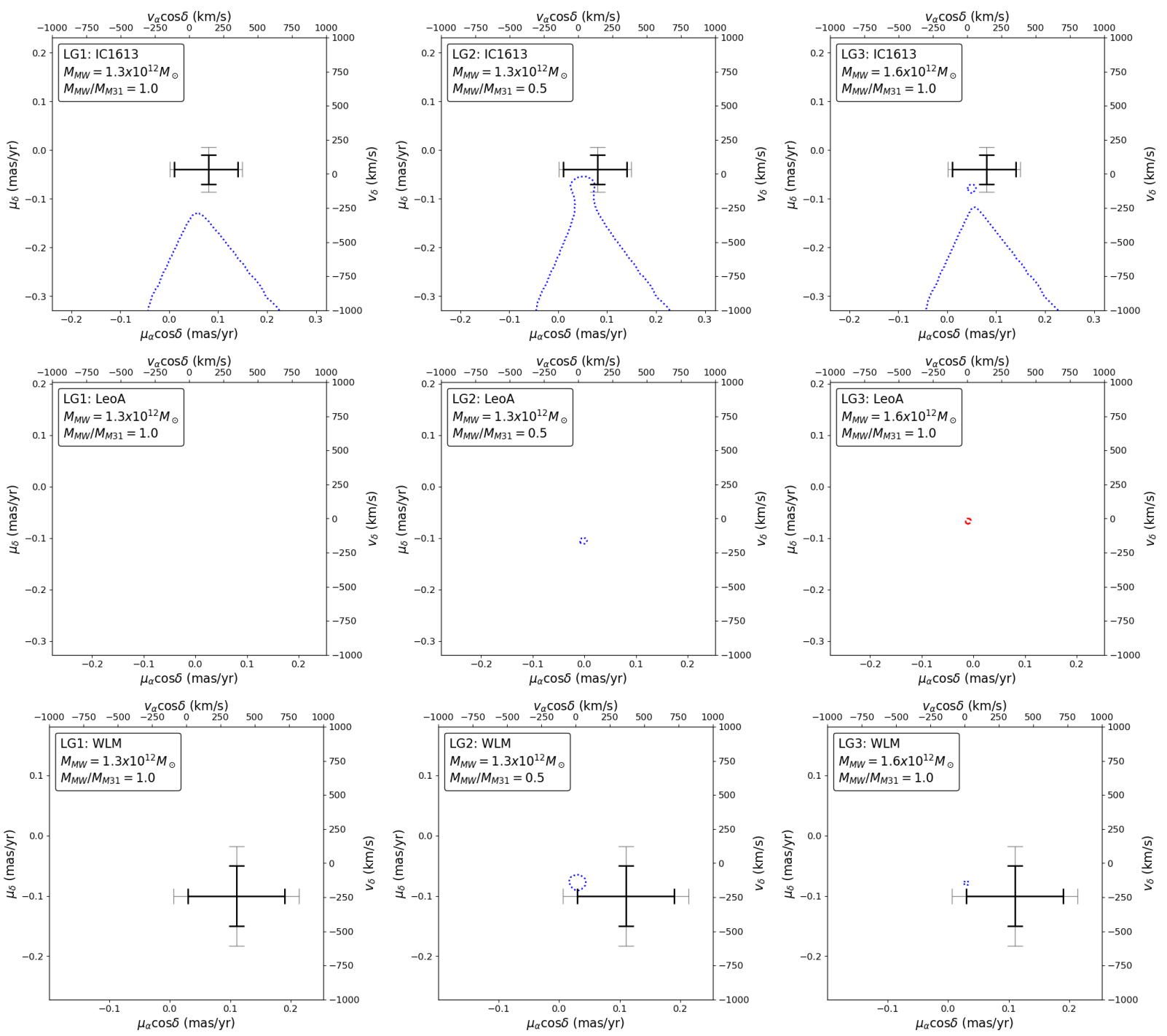

Figure 5. Same as Figure 4, for IC 1613, Leo A and WLM. Note that the measured systemic proper motion of Leo A falls outside of the proper motion space explored (although it is consistent with zero at less than $2 \sigma$ ).

$(\sim 200-240)$, and Phoenix has more than 3 times as many (more than 300 , on average);

- NGC 6822: In principle, NGC 6822 could have had interactions with either the Milky Way or M31, but the relatively precise proper motion measurement means that an M31 interaction is unlikely at more than $4-\sigma$. A Milky Way interaction requires NGC 6822 to have a Galactocentric tangential velocity of less than $\sim 110 \mathrm{~km} \mathrm{~s}^{-1}$, and this is at the edge of the $1-\sigma$ proper motion uncertainties. It is worth noting the importance of systematic errors for NGC 6822 , since these contribute more than random uncertainties. We examine the orbit of NGC 6822 in more detail in the next section;

- IC 1613: The current proper motion measurement of IC 1613 is inconsistent at approximately $2-\sigma$ with ever having an interaction with M31, under the assumption that M31's mass is only around $1.3 \times 10^{12} M_{\odot}$ (as in LG1). The region of parameter space in which an interaction can occur corresponds broadly to IC 1613's current space velocity pointing away from M31, and aligned within $\sim 40$ degrees of the radial vector from M31. For a more massive M31 (as in LG2 and LG3), then the proper motion of IC 1613 more closely overlaps with the regime of parameter space in which interactions with M31 can occur;

- Leo A: There is only a tiny regime of parameter space in which Leo A could have interacted with either the Milky Way or M31. For the latter, M31 must be massive (as in LG2; around twice the most likely mass for the Milky Way). For the former, the Milky Way must also be relatively massive (as in LG3; at the upper end of the current preferred mass range) and the relative tangential velocity of the two must be less than around $40 \mathrm{~km} \mathrm{~s}^{-1}$. Like Leo T, the current estimate for the proper motion of Leo A is completely useless for distinguishing these scenarios, but the relatively tiny regions of parameter space that need to be excluded means that future improvements in the size of the uncertainities associated with the proper motion may put meaningful constraints on the likelihood or otherwise of a backspash origin; $\mathrm{x}$

- WLM: Within the setups explored, this dwarf can only have interacted with M31, and can only have done so if M31 is relatively massive (as in LG2 and LG3). Those orbits which lead to an interaction require WLM to be close to apocenter, and with relative velocities between the two less than $\sim 60 \mathrm{~km} \mathrm{~s}^{-1}$. This region 
of parameter space overlaps with our current estimate of its proper motion.

\subsection{The orbit of NGC 6822}

While several of the galaxies listed in Table 2 have good proper motion measurements in an angular sense (better than a few tenths of a mas/yr), only NGC 6822 has a tangential velocity constrained to much better than $100 \mathrm{~km} \mathrm{~s}^{-1}$ or so (even after systematic uncertainities are taken into account). Thus, compared to any of the other galaxies in Figures 4 and 5, the volume of likely parameter space allowed by the proper motion measurement of NGC 6822 is considerably smaller than the volume of parameter space probed by our previous grid search. As such, we decided to conduct a more detailed analysis of the possible orbits of NGC 6822 within the Local Group.

Figure 6 shows the orbital path of NGC 6822 in the Local Group over the last 10 Gyrs, for the LG1 realisation. The first three panels show projections in the $x-y-z$ space, and the fourth panel shows the separation of the dwarf as a function of time from the Milky Way and M31 (red and blue lines, respectively). Solid points indicate the current positions of the three galaxies. The coordinates are based on the Galactocentric frame, but rotated so that M31 lies on the $\mathrm{x}$ axis, and centered on the midpoint of the Milky Way - M31 positions. Here, we have set the radial velocity, proper motion and distance of M31 to the values used earlier. We have also set the radial velocity and distance of NGC 6822 to the values used earlier, and we have set the proper motion of NGC 6822 to the measured value. In this idealised scenario, NGC 6822 is clearly on its first infall into the Local Group and has had no interaction with either of the big galaxies. For most of its history, it has been falling nearly radially into the Local Group, and 10 Gyrs ago it was approximately $1.9 \mathrm{Mpc}$ away from the Milky Way (3.3 Mpc away from M31).

To better understand the range of orbits consistent with the observational parameters, we use the three Local Group setups, with the different values of $M_{M W}$ and $f$, described previously. For each setup, $10^{5}$ realisations of the Milky Way, M31 and NGC 6822 are evolved backwards in time for 10 Gyrs. For each realisation, values for the distance modulus, the heliocentric radial velocity and the proper motion of both NGC 6822 and M31 are selected from Gaussian distributions. These are centered on the relevant measured values and have a standard deviation equal to the uncertainty on the measured value.

Out of the three sets of $10^{5}$ realisations, only eight orbits are found that involve NGC 6822 passing within $300 \mathrm{kpc}$ of M31, in line with our findings from the grid search, and essentially ruling out a past M31 interaction for NGC 6822. In constrast, 6-9\% of the realisations involve $\mathrm{a} \leq 300 \mathrm{kpc}$ passage between NGC 6822 and the Milky Way. This too is broadly in line with the results from the grid search, which did not take into account the uncertainties in all the other measured parameters for NGC 6822 and M31.

More specifically, for each set of $10^{5}$ realisations:

(i) LG1: $6631 \pm 81$ orbits $(6.6 \%)$ pass within $300 \mathrm{kpc}$ of the Milky Way; 1 orbit passes within $300 \mathrm{kpc}$ of the M31 (uncertainties are Poisson statistics);

(ii) LG2: $6491 \pm 81$ orbits (6.5\%) pass within $300 \mathrm{kpc}$ of the Milky Way; 6 orbits pass within $300 \mathrm{kpc}$ of the M31;

(iii) LG3: $8298 \pm 91$ orbits $(8.3 \%)$ pass within $300 \mathrm{kpc}$ of the Milky Way; 1 orbit passes within $300 \mathrm{kpc}$ of the M31.

We conclude from this analysis that the current uncertainties on the relevant observational parameters imply that NGC 6822 has not had any interactions with M31 or the Milky Way, at 99.99\% confidence and better than $90 \%$ confidence, respectively. We also note that the mass of M31 does not have any significant impact on the likelihood that NGC 6822 is a backsplash galaxy or not.

Given that NGC 6822 appears to be on its first infall into the Local Group, a natural question to ask is "infall from where?". Figure 7 shows a contour map of the predicted positions of NGC 682210 Gyrs ago, in celestial coordinates, for those orbits in which NGC 6822 did not pass within $300 \mathrm{kpc}$ of the Milky Way. The LG1 realisation was used to make this figure, although all three realisations are qualitatively similar. Further, the distribution of points is similar whether the plot is made for $10 \mathrm{Gyrs}$ ago or $8 \mathrm{Gyrs}$ ago, as is expected given that at these early times NGC 6822 is far from the Galaxy and falling mostly radially towards it. $2 \times 2$ degree pixels were used, and the counts per pixel were weighted by $\cos \delta$. Counts were smoothed by a Gaussian with $\sigma=1$ pixel. Contour levels are set at 2, 5, 10, 30, $70,80,95$ and $98 \%$ of the maximum value.

Also shown in Figure 7 are the (current) locations of galaxies in nearby groups from Karachentsev (2005). The closest of these groups (Maffei) is approximately $3 \mathrm{Mpc}$ away, and their projected positions on the sky should not have shifted significantly over the last 10 Gyrs given that mostly radial motion relative to the Local Group is expected. Note that the very nearby NGC3109 galaxy group is not shown. Its very close proximity to the Local Group means that it is quite possible that it and the Local Group have undergone a more complex, non-radial orbit. This means that the present location of this galaxy group ( $\alpha \sim 151^{\circ}, \delta \sim-26^{\circ}$ ) on the sky is not necessarily a good indicator of its historic position. It is well known that all the closest galaxy groups are found along a great circle path, indicative of the Local Group being embedded in a "local sheet" of nearby structures (e.g., Tully et al. 2008 and references therein).

There is clearly a reasonably-well defined locus on the sky from which NGC 6822 likely originated. This locus is assiduous in avoiding any overlap with the locations of any of the nearest galaxy groups. This is perhaps to be expected: should NGC 6822 have originated in a dark matter halo in the vicinity of any of the other galaxy groups, then it would likely have ended up as a satellite in one of these groups. So this appears consistent with the idea that NGC 6822 formed in relative isolation in the periphery of the structure now known as the Local Group of galaxies, close enough that the gravity of the Local Group won out over the Hubble expansion. Over the course of a Hubble time, it has fallen in to the Local Group, and is now in relatively close proximity to the Milky Way. We note that there have been suggestions that NGC6822 may have undergone a recent interaction with another small stellar system (e.g., see de Blok \& Walter 2000; Demers et al. 2006, but see also Cannon et al. 2012; Thompson et al. 2016), in which case perhaps NGC 6822 had a dwarf-like companion at earlier times.

And what of the immediate future of NGC 6822? Integrating forward the orbital realisations from LG1 for which there was not a previous interaction suggests that it has only a $0.04 / 0.6 \%$ chance of having an interaction (i.e., passing within $\sim 300 \mathrm{kpc}$ ) with the Milky Way/M31 in the next 4 Gyrs. But this uneventful future for NGC 6822 is not shared by the Milky Way and M31. For in about 4 Gyrs, these two large galaxies will be within about $100 \mathrm{kpc}$ of each other, in the midst of a near head-on collision (e.g., see van der Marel et al. 2012b). The most likely fate for NGC 6822 over the next 4 Gyrs is shown with the dotted line in Figure 6. In this realisation, NGC 6822 is currently near pericenter with the Milky Way, and will continue on its relatively uneventful path through space, oblivious to the imminent formation of the massive galaxy formerly known as the Local Group. 

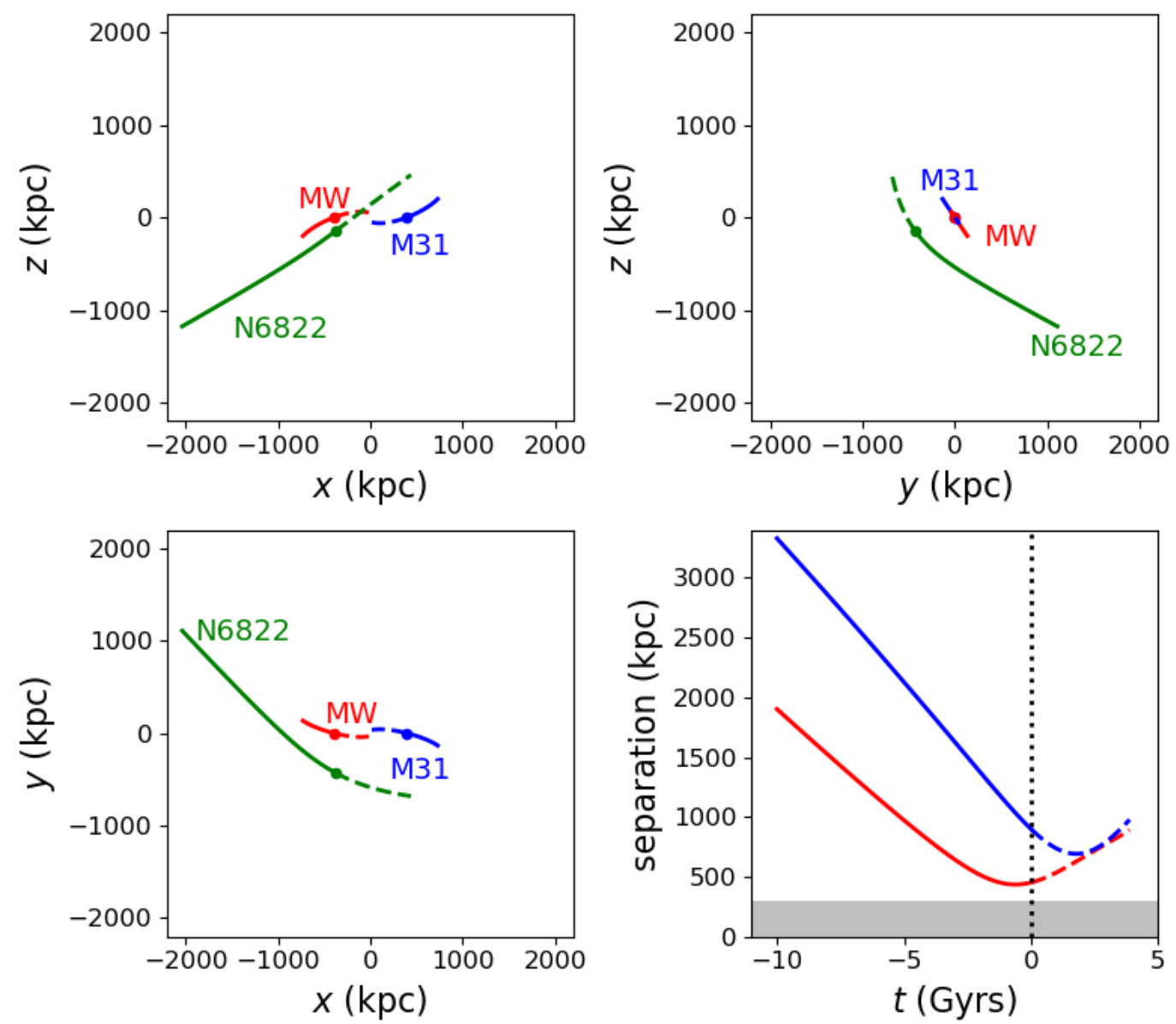

Figure 6. First three panels show the projection of the most likely orbit of NGC 6822 (green) relative to the Milky Way (red) and M31 (blue) over the last 10 Gyrs (solid lines), and for the next 4 Gyrs (dashed lines), using the LG1 potential. Solid points indicate current positions of the galaxies. The coordinate system is centered on the mid point of the Milky Way - M31 positions, and aligned so that the x-axis connects the Milky Way and M31. The fourth panel shows the separation of the dwarf as a function of time from the Milky Way (red line) and M31 (blue line). Present day is indicated by the dotted line. The grey shaded region corresponds to a separation of less than $300 \mathrm{kpc}$ from either large galaxy

\section{DISCUSSION AND SUMMARY}

\subsection{Proper motion measurements and future Gaia data releases}

The parameter space probed in Figures 4 and 5 gives a good handle on the current usefulness of the orbital constraints for the dwarf galaxies provided by the new measurements of their proper motions. From these considerations, and our orbital analysis, we conclude that the proper motion measurement of NGC 6822 in Table 2 is interesting, even including systematic uncertainties. IC 1613 and WLM both have good measurements of their proper motions in an angular sense, but given their distances, their tangential velocities are less interesting. The current proper motion measurement for the remaining galaxies are not particularly useful. While smaller uncertainties are needed, we note that the currently derived proper motions are generally within $2-\sigma$ of the predicted proper motions by Shaya \& Tully (2013).

It is important to recognise the impact future Gaia data releases will have on these measurements. Firstly, it is unlikely that any of the galaxies listed in Table 1 but not in Table 2 will ever have any meaningful measurements of their proper motion using Gaia data: there are simply not enough bright stars visible to Gaia in these galaxies. Secondly, random errors in all these measurements will decrease significantly as the baseline $(t)$ of the Gaia observations increases and the signal to noise of the observations increases, leading to a proportionality of $t^{-3 / 2}$. For example, the imminent release of Gaia Data Release 3 in December 2020 will be based on 34 months of observations compared to 22 months for Data Release 2, and proper motions are expected to be a factor of 2 better than DR2 4 . This should mean that the tangential velocities of IC 1613, WLM and possibly Phoenix will be able to be constrained at an interesting level, especially if systematic uncertainties also decrease. By the end of the complete mission in 2022, with an 8 year baseline, it seems likely that the random uncertainties on the tangential velocities should decrease by a factor of 9 in comparison to these DR 2 results. In this case, all the galaxies in Table 2 except possibly Leo $\mathrm{T}$ and Leo A should have uncertainties in their tangential velocities of several tens of $\mathrm{km} \mathrm{s}^{-1}$ in each direction or better. By this point, the velocity components of NGC 6822 should be measured with exquisite precision.

\subsection{Non-Gaia proper motions}

So what of Leo T, Leo A, and the Solo dwarfs listed in Table 1 that do not have (m)any stars visible in Gaia? More sensitive observatories

\footnotetext{
${ }^{4}$ https://www.cosmos.esa.int/web/gaia/earlydr3
} 


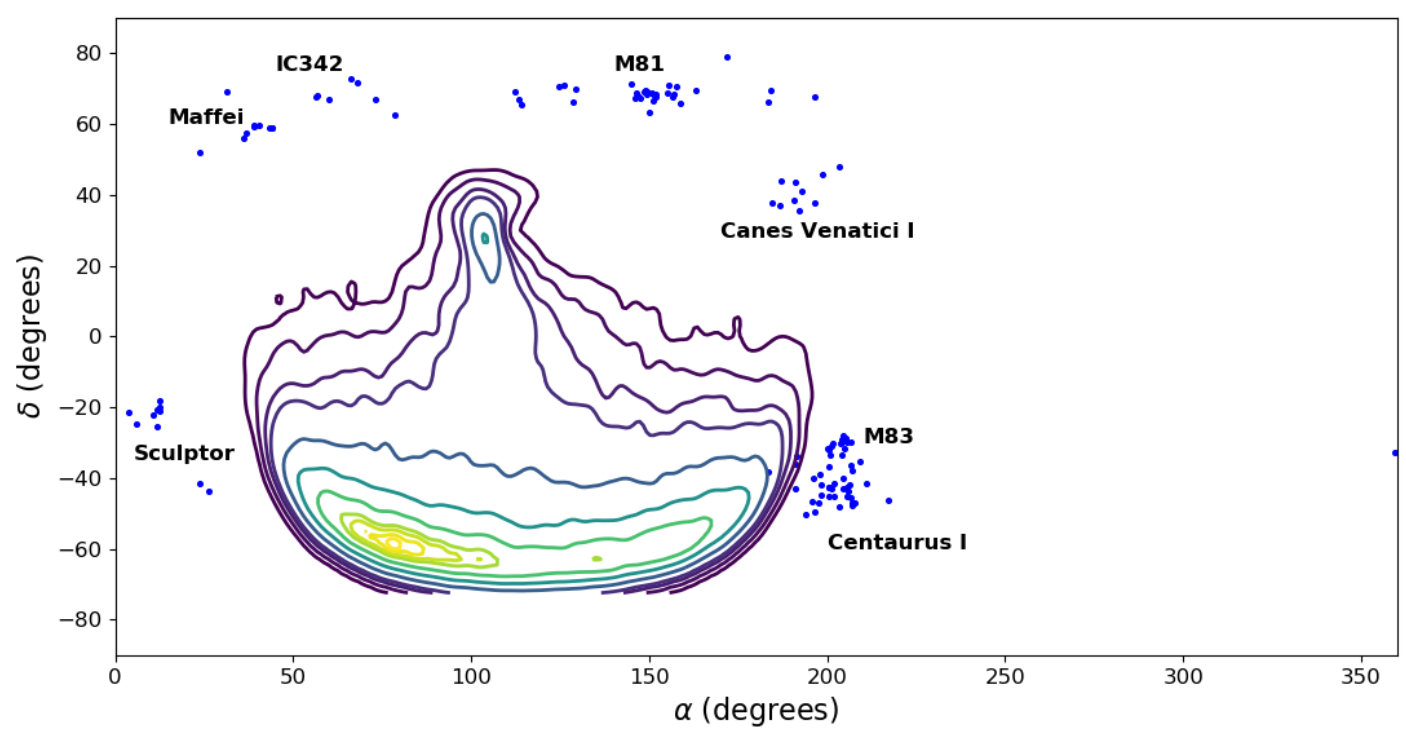

Figure 7. Contour map of the predicted positions of NGC 682210 Gyrs ago, in celestial coordinates, for those orbits in which NGC 6822 did not pass within $300 \mathrm{kpc}$ of the Milky Way, in the LG1 realisation. $2 \times 2$ degree pixels were used, and the counts per pixel were weighted by cos $\delta$. Counts were smoothed by a Gaussian with $\sigma=1$ pixel. Contour levels are set at 2, 5, 10, 30, 70, 80, 95 and 98\% of the maximum value. Also shown are the (current) locations of galaxies in nearby groups from Karachentsev et al. (2005).

are clearly required to detect a sufficient number of their stars, but combining sensitivity with high spatial resolution and low systematic errrors means there are only a few facilities in the optical and IR regimes that could make these measurements in the next decade.

There are many large aperture facilities on the ground. Most notably, the Legacy Survey for Space and Time, to be conducted by the Vera C. Rubin Observatory, will be conducted over a period of a decade. Its repeated observations of the entire southern hemisphere will make it very suitable for proper motion studies, and its data acquisition strategy and heavy focus on data processing will likely ensure a good understanding of systematic errors. However, as a seeing-limited facility, its spatial resolution will likely not generally be sufficient for many proper motion studies beyond the Milky Way sub-group. In comparison, the very high spatial resolution of the Thirty Meter Telescope (TMT) and the Extremely Large Telescope (ELT) equipped with multi-conjugate adaptive optics systems, will certainly provide very high spatial resolution (e.g., see Evslin 2015 for the science that can be enabled). Here, however, the challenge will be to obtain a sufficiently detailed understanding and characterisation of the systematic uncertainties which will otherwise dominate, and which are contributed to by residual spatial distortions from the adaptive optics system (e.g., see Taheri et al., 2020, submitted, and references therein).

Space-based observatories will therefore provide the most promising avenue for more Local Group proper motions. It is worth highlighting the proven utility of HST for these types of measurements. HST has been used to conduct proper motion studies of dwarf galaxies for many years, with early ground-breaking work that utilised background quasars to define the absolute reference frame (e.g., Piatek et al. 2002, 2003, 2005, 2006, 2007; Kallivayalil et al. 2006a,b, 2013). Later adoption of a reference frame defined by background galaxies reduced the restriction in field selection caused by the requirement for there to be a background quasar. Since then, proper motion measurements beyond the Milky Way subgroup have been made for M31 (Sohn et al. 2012), NGC147 and NGC185 (Sohn et al. 2020). Proper motion measurements also exist for M33 and IC10 from VLBI observations (Brunthaler et al. 2005, 2007). We note that Gaia DR2 has also been used to independently obtain the proper motions of M31 and M33 (van der Marel et al. 2019), although at these distances the Gaia DR2 accuracy is significantly less than for HST (and, of course, VLBI).

Upcoming space missions in the next decade with the potential to provide new Local Group proper motion measurements include the James Webb Space Telescope (JWST), the Nancy Grace Roman Space Telescope, and the Euclid space mission. These observatories will all be active by mid-decade, and all operate at longer wavelengths than HST. Taking into account their apertures, their spatial resolutions will either be similar or slightly poorer than HST (but still very good in an absolute sense). However, a major advantage of HST for proper motion studies is the very long baseline of observations that exist given the maturity of this facility. In this regard, it is very likely that, even using data on these dwarfs from JWST, Roman or Euclid, first epoch observations will be from HST. Indeed, programs are already underway with HST to obtain first epoch observations of Local Group dwarf galaxies where none currently exist, or to obtain second-epoch observations where first-epoch already exist. Significant survey datasets in this regard include the Advanced Camera for Surveys Local Cosmology from Isolated Dwarfs (ACS LCID) survey for isolated dwarfs (e.g., Cole et al. 2007; Monelli et al. 2010a,b; Hidalgo et al. 2011; Skillman et al. 2014), the Initial Star formation and Lifetimes of Andromeda Satellites (ISLANDS) for M31 dwarf spheroidals (Monelli et al. 2016; Skillman et al. 2017; Martínez-Vázquez et al. 2017), and work on fainter members of the M31 satellite system (Martin et al. 2017; Weisz et al. 2019).

\subsection{Summary}

In this paper, we have measured systemic proper motions for distant $(d \sim 450-900 \mathrm{kpc})$ dwarf galaxies in the Local Group using CFHT and Gaia Data Release 2 and investigated if these (currently) isolated galaxies have ever had an interaction with either the Milky Way or M31. We find that NGC 6822, Leo A, IC 1613 and WLM 
have a sufficient number of bright stars with reliable astrometry to derive proper motions for these four galaxies. We apply a variant of the methodology described in MV2020 to obtain estimates of their systemic proper motions. The results for NGC 6822, IC 1613 and WLM are good in an angular sense - systematic errors in Gaia DR2 proper motions either dominate or are major contributers to the error budgets. However, the large distances of IC 1613 and WLM mean that the resulting constraint on their orbits are still relatively weak.

We explore the orbital parameter space for these isolated galaxies in light of these new measurement, and also include Leo T, Eridanus 2 and Phoenix, which have recent proper motion estimates from MV2020. We conclude that Phoenix, Leo A and WLM are unlikely to have ever interacted with the Milky Way or M31, unless these galaxies are very massive (at the upper end of any recent estimates of their masses). We rule out a past interaction of NGC 6822 with M31 at $\sim 99.99 \%$ confidence, and find there is less than a $10 \%$ chance that NGC 6822 has had an interaction with the Milky Way. We also explore the origins of this galaxy in the periphery of the young Local Group. For the remaining dwarfs under consideration, the current proper motions do not yet rule out the possibility that these galaxies may be backsplash systems. However, our results indicate that future data releases from Gaia will provide good or excellent constraints on the interaction history of these seven galaxies, with the possible exceptions of Leo A and Leo T. These last two galaxies suffer from a combination of only a few bright stars being visible to Gaia, and a relative dearth of visits by the spacecraft as a result of the Gaia scanning law.

\section{ACKNOWLEDGEMENTS}

We thank the anonymous referee for comments that improved the paper. We also thank Matías Blaña and Tony Sohn for their comments.

AWM and KAV would like to acknowledge funding from the National Science and Engineering Research Council Discovery Grants program. GB acknowledges financial support through the grants (AEI/FEDER, UE) AYA2017-89076-P, as well as by the Ministerio de Ciencia, Innovación y Universidades (MCIU), through the State Budget and by the Consejer a de Economia, Industria, Comercio y Conocimiento of the Canary Islands Autonomous Community, through the Regional Budget.

Based on observations obtained with MegaPrime/MegaCam, a joint project of CFHT and CEA/DAPNIA, at the Canada-FranceHawaii Telescope (CFHT) which is operated by the National Research Council (NRC) of Canada, the Institut National des Science de l'Univers of the Centre National de la Recherche Scientifique (CNRS) of France, and the University of Hawaii. The observations at the Canada-France-Hawaii Telescope were performed with care and respect from the summit of Maunakea which is a significant cultural and historic site.

\section{DATA AVAILABILITY}

Some of the data underlying this article were accessed from the Gaia Archive (https://gea.esac.esa.int/archive/). The CFHT data underlying this article are available through the CFHT archive hosted by the Canadian Astronomy Data Center (http: //www. cadc-ccda.hia-iha.nrc-cnrc.gc.ca/en/cfht/). Derived data products will be shared on reasonable request to the corresponding author.

\section{REFERENCES}

Blaña M., Burkert A., Fellhauer M., Schartmann M., Alig C., 2020, MNRAS, 497, 3601

Bland-Hawthorn J., Gerhard O., 2016, ARA\&A, 54, 529

Boselli A., Cortese L., Boquien M., Boissier S., Catinella B., Gavazzi G., Lagos C., Saintonge A., 2014, A\&A, 564, A67

Bouchard A., Da Costa G. S., Jerjen H., 2009, AJ, 137, 3038

Boulade O., et al., 2003, in Iye M., Moorwood A. F. M., eds, Proc. SPIEVol. 4841, Instrument Design and Performance for Optical/Infrared Groundbased Telescopes. pp 72-81, doi:10.1117/12.459890

Brunthaler A., Reid M. J., Falcke H., Greenhill L. J., Henkel C., 2005, Science, 307, 1440

Brunthaler A., Reid M. J., Falcke H., Henkel C., Menten K. M., 2007, A\&A, 462,101

Buck T., Macciò A. V., Dutton A. A., Obreja A., Frings J., 2019, MNRAS, 483,1314

Cannon J. M., et al., 2012, ApJ, 747, 122

Cautun M., et al., 2020, MNRAS, 494, 4291

Cole A. A., et al., 2007, ApJ, 659, L17

Davidge T. J., McConnachie A. W., Fardal M. A., Fliri J., Valls-Gabaud D., Chapman S. C., Lewis G. F., Rich R. M., 2012, ApJ, 751, 74

Dehnen W., 2000, ApJ, 536, L39

Demers S., Battinelli P., Kunkel W. E., 2006, ApJ, 636, L85

Dohm-Palmer R. C., Skillman E. D., Mateo M., Saha A., Dolphin A., Tolstoy E., Gallagher J. S., Cole A. A., 2002, AJ, 123, 813

Dressler A., et al., 2011, PASP, 123, 288

Drlica-Wagner A., et al., 2020, ApJ, 893, 47

Eadie G., Jurić M., 2019, ApJ, 875, 159

Einasto J., Saar E., Kaasik A., Chernin A. D., 1974, Nature, 252, 111

Evslin J., 2015, MNRAS, 452, L41

Fardal M. A., et al., 2013, MNRAS, 434, 2779

Foreman-Mackey D., Hogg D. W., Lang D., Goodman J., 2013, PASP, 125, 306

Foreman-Mackey D., et al., 2019, The Journal of Open Source Software, 4, 1864

Fritz T. K., Battaglia G., Pawlowski M. S., Kallivayalil N., van der Marel R., Sohn S. T., Brook C., Besla G., 2018, A\&A, 619, A103

Fritz T. K., Carrera R., Battaglia G., Taibi S., 2019, A\&A, 623, A129

Gaia Collaboration et al., 2018a, A\&A, 616, A1

Gaia Collaboration et al., 2018b, A\&A, 616, A12

Geha M., Blanton M. R., Yan R., Tinker J. L., 2012, ApJ, 757, 85

Gill S. P. D., Knebe A., Gibson B. K., 2005, MNRAS, 356, 1327

Girardi L., Bertelli G., Bressan A., Chiosi C., Groenewegen M. A. T., Marigo P., Salasnich B., Weiss A., 2002, A\&A, 391, 195

Gravity Collaboration et al., 2018, A\&A, 615, L15

Green G., 2018, The Journal of Open Source Software, 3, 695

Hammer F., Puech M., Chemin L., Flores H., Lehnert M. D., 2007, ApJ, 662, 322

Hidalgo S. L., et al., 2011, ApJ, 730, 14

Higgs C. R., et al., 2016, MNRAS, 458, 1678

Higgs C. R., et al., 2020

Ibata R., Chapman S., Ferguson A. M. N., Lewis G., Irwin M., Tanvir N., 2005, ApJ, 634, 287

Irwin M. J., et al., 2007, ApJ, 656, L13

Jacobs B. A., Tully R. B., Rizzi L., Karachentsev I. D., Chiboucas K., Held E. V., 2011, AJ, 141, 106

Kallivayalil N., van der Marel R. P., Alcock C., Axelrod T., Cook K. H., Drake A. J., Geha M., 2006a, ApJ, 638, 772

Kallivayalil N., van der Marel R. P., Alcock C., 2006b, ApJ, 652, 1213

Kallivayalil N., van der Marel R. P., Besla G., Anderson J., Alcock C., 2013, ApJ, 764, 161

Kallivayalil N., et al., 2018, ApJ, 867, 19

Karachentsev I. D., 2005, AJ, 129, 178

Kawata D., Mulchaey J. S., 2008, ApJ, 672, L103

Kazantzidis S., Łokas E. L., Callegari S., Mayer L., Moustakas L. A., 2011, ApJ, 726, 98

Klypin A., Zhao H., Somerville R. S., 2002, ApJ, 573, 597 
Lindegren L., et al., 2018, A\&A, 616, A2

Longeard N., et al., 2018, MNRAS, 480, 2609

Longeard N., et al., 2020a, arXiv e-prints, p. arXiv:2005.05976

Longeard N., et al., 2020b, MNRAS, 491, 356

Martin N. F., et al., 2017, ApJ, 850, 16

Martínez-Vázquez C. E., et al., 2017, ApJ, 850, 137

Massari D., Helmi A., 2018, A\&A, 620, A155

Mau S., et al., 2020, ApJ, 890, 136

Mayer L., Mastropietro C., Wadsley J., Stadel J., Moore B., 2006, MNRAS, 369,1021

McConnachie A. W., 2012, AJ, 144, 4

McConnachie A. W., Venn K. A., 2020, AJ, 160, 124

McConnachie A. W., Irwin M. J., Ferguson A. M. N., Ibata R. A., Lewis G. F., Tanvir N., 2005, MNRAS, 356, 979

McConnachie A. W., et al., 2018, ApJ, 868, 55

McLeod B., et al., 2015, Publications of the Astronomical Society of the Pacific, 127, 366

Monelli M., et al., 2010a, ApJ, 720, 1225

Monelli M., et al., 2010b, ApJ, 722, 1864

Monelli M., et al., 2016, ApJ, 819, 147

Mori M., Burkert A., 2000, ApJ, 538, 559

Muñoz R. R., Côté P., Santana F. A., Geha M., Simon J. D., Oyarzún G. A., Stetson P. B., Djorgovski S. G., 2018, ApJ, 860, 66

Pace A. B., Li T. S., 2019, ApJ, 875, 77

Patel E., Besla G., Mandel K., 2017, MNRAS, 468, 3428

Peñarrubia J., Navarro J. F., McConnachie A. W., 2008, ApJ, 673, 226

Peñarrubia J., Ma Y.-Z., Walker M. G., McConnachie A., 2014, MNRAS, 443, 2204

Piatek S., et al., 2002, AJ, 124, 3198

Piatek S., Pryor C., Olszewski E. W., Harris H. C., Mateo M., Minniti D., Tinney C. G., 2003, AJ, 126, 2346

Piatek S., Pryor C., Bristow P., Olszewski E., Harris H., Mateo M., Minniti D., Tinney C., 2005, astro-ph/0503620,

Piatek S., Pryor C., Bristow P., Olszewski E. W., Harris H. C., Mateo M., Minniti D., Tinney C. G., 2006, AJ, 131, 1445

Piatek S., Pryor C., Bristow P., Olszewski E. W., Harris H. C., Mateo M., Minniti D., Tinney C. G., 2007, AJ, 133, 818

Posti L., Helmi A., 2019, A\&A, 621, A56

Schlafly E. F., Finkbeiner D. P., 2011, ApJ, 737, 103

Schlegel D. J., Finkbeiner D. P., Davis M., 1998, ApJ, 500, 525

Schönrich R., Binney J., Dehnen W., 2010, MNRAS, 403, 1829

Shaya E. J., Tully R. B., 2013, MNRAS, 436, 2096

Simon J. D., 2018, ApJ, 863, 89

Simon J. D., et al., 2020, ApJ, 892, 137

Skillman E. D., et al., 2014, ApJ, 786, 44

Skillman E. D., et al., 2017, ApJ, 837, 102

Sohn S. T., Anderson J., van der Marel R. P., 2012, ApJ, 753, 7

Sohn S. T., Patel E., Fardal M. A., Besla G., van der Marel R. P., Geha M., Guhathakurta P., 2020, arXiv e-prints, p. arXiv:2008.06055

Teyssier M., Johnston K. V., Kuhlen M., 2012, MNRAS, 426, 1808

Thompson G. P., Ryan S. G., Sibbons L. F., 2016, MNRAS, 462, 3376

Tully R. B., Shaya E. J., Karachentsev I. D., Courtois H. M., Kocevski D. D., Rizzi L., Peel A., 2008, ApJ, 676, 184

Venn K. A., et al., 2001, ApJ, 547, 765

Venn K. A., Tolstoy E., Kaufer A., Skillman E. D., Clarkson S. M., Smartt S. J., Lennon D. J., Kudritzki R. P., 2003, AJ, 126, 1326

Vollmer B., Marcelin M., Amram P., Balkowski C., Cayatte V., Garrido O., 2000, A\&A, 364, 532

Watkins L. L., Evans N. W., An J. H., 2010, MNRAS, 406, 264

Weisz D. R., Dolphin A. E., Skillman E. D., Holtzman J., Gilbert K. M., Dalcanton J. J., Williams B. F., 2014, ApJ, 789, 147

Weisz D. R., et al., 2019, MNRAS, 489, 763

Whiting A. B., Irwin M. J., Hau G. K. T., 1997, AJ, 114, 996

Whiting A. B., Hau G. K. T., Irwin M., 1999, AJ, 118, 2767

Whiting A. B., Hau G. K. T., Irwin M., Verdugo M., 2007, AJ, 133, 715

de Blok W. J. G., Walter F., 2000, ApJ, 537, L95

van den Bergh S., 2000, The Galaxies of the Local Group. Cambridge van der Marel R. P., Fardal M., Besla G., Beaton R. L., Sohn S. T., Anderson J., Brown T., Guhathakurta P., 2012a, ApJ, 753, 8

van der Marel R. P., Besla G., Cox T. J., Sohn S. T., Anderson J., 2012b, ApJ, 753,9

van der Marel R. P., Fardal M. A., Sohn S. T., Patel E., Besla G., del Pino A., Sahlmann J., Watkins L. L., 2019, ApJ, 872, 24 